\title{
Study of Some Parameters of Modified Chaplygin Gas in Galileon Gravity Theory from Observational Perspective
}

\author{
Chayan Ranjit1 \\ Department of Mathematics, Seacom Engineering College, Howrah-711 302, India.
}

\begin{abstract}
Prabir Rudra2
Department of Mathematics, Pailan College of Management and Technology, Bengal Pailan Park, Kolkata-700 104, India.

Ujjal Debnath 3

Department of Mathematics, Indian Institute of Engineering Science and Technology, Shibpur, Howrah-711 103, India.
\end{abstract}

\begin{abstract}
We have assumed the FRW model of the universe in Galileon gravity, which is filled with dark matter and Modified Chaplygin gas (MCG) type dark energy. We present the Hubble parameter in terms of some unknown parameters and observational parameters with the redshift $z$. Some cosmological parameters are reconstructed and plots are generated to study the nature of the model and its viability. It is seen that the model is perfectly consistent with the present cosmic acceleration. From observed Hubble data (OHD) set or Stern data set of 12 points, we have obtained the bounds of the arbitrary parameters $(A, B) \&(A, C)$ by minimizing the $\chi^{2}$ test. Next due to joint analysis of Stern $+B A O$ and Stern $+B A O+C M B$ observations, we have also obtained the best fit values and the bounds of the parameters $(A, B) \&(A, C)$ by fixing some other parameters. The best-fit values and bounds of the parameters are obtained with $66 \%, 90 \%$ and $99 \%$ confidence levels for Stern, Stern $+B A O$ and Stern $+B A O+C M B$ joint analysis. Next we have also taken type Ia supernovae data set (union 2 data set with 557 data points). The distance modulus $\mu(z)$ against redshift $z$ for our theoretical MCG model in Galileon gravity have been tested for the best fit values of the parameters and the observed SNe Ia union 2 data sample and from this, we have concluded that our model is in agreement with the union 2 sample data.
\end{abstract}

\section{Introduction}

Observational evidence strongly points to an accelerated expansion of the Universe, but the physical origin of this acceleration is still unknown. The observations include type Ia Supernovae and Cosmic Microwave Background (CMB) [1, 2, 3, 4, 5, radiation. The standard explanation invokes an unknown "dark energy" component which has the property that positive energy density and negative pressure. Observations indicate that dark energy occupies about $70 \%$ of the total energy of the universe, and the contribution of dark matter is $\sim 26 \%$. This accelerated expansion of the universe has also been strongly confirmed by some other independent experiments like Sloan Digital Sky Survey (SDSS) [6], Baryonic Acoustic Oscillation (BAO) [7, WMAP data analysis 8, 9] etc. Over the past decade, there have been many theoretical models for mimicking the dark energy behaviors, such as the simplest (just) cosmological constant in which the equation of state is independent of the cosmic time and which can fit the observations well. This model is the so-called $\Lambda \mathrm{CDM}$, containing

\footnotetext{
${ }^{1}$ chayanranjit@gmail.com

${ }^{2}$ prudra.math@gmail.com

${ }^{3}$ ujjaldebnath@gmail.com
} 
a mixture of cosmological constant $\Lambda$ and cold dark matter (CDM). However, two problems arise from this scenario, namely "fine-tuning" and the "cosmic coincidence" problems. In order to solve these two problems, many dynamical dark energy models were suggested, whose equation of state evolves with cosmic time. The scalar field or quintessence [10, 11] is one of the most favored candidate of dark energy which produce sufficient negative pressure to drive cosmic acceleration. In order to alleviate the cosmological-constant problems and explain the acceleration expansion, many dynamical dark energy models have been proposed, such as K-essence, Tachyon, Phantom, quintom, Chaplygin gas model, etc [12, 13, 14, 15, 16]. Also the interacting dark energy models including Modified Chaplygin gas [17, holographic dark energy model [18, and braneworld model [19] have been proposed. Recently, based on principle of quantum gravity, the agegraphic dark energy (ADE) and the new agegraphic dark energy (NADE) models were proposed by Cai 20] and Wei et al 21] respectively. The theoretical models have been tally with the observations with different data sets say TONRY, Gold sample data sets [22, 3, 23, 24 etc. In Einstein's gravity, the modified Chaplygin gas [17] best fits with the 3 year WMAP and the SDSS data with the choice of parameters $A=0.085$ and $\alpha=1.724$ [25] which are improved constraints than the previous ones $-0.35<A<0.025$ [26].

Another possibility is that general relativity is only accurate on small scales and has to be modified on cosmological distances. One of these is modified gravity theories. In this case, cosmic acceleration would arise not from dark energy as a substance but rather from the dynamics of modified gravity. Modified gravity constitutes an interesting dynamical alternative to $\Lambda \mathrm{CDM}$ cosmology in that it is also able to describe the current acceleration in the expansion of our universe. One of the simplest modified gravity is DGP brane-world model 27. The other alternative approach dealing with the acceleration problem of the Universe is changing the gravity law through the modification of action of gravity by means of using $f(R)$ gravity [28, 29] instead of the Einstein-Hilbert action. Some of these models, such as $1 / R$ and logarithmic models, provide an acceleration for the Universe at the present time [30. Other modified gravity includes $f(T)$ gravity, $f(G)$ gravity, Gauss-Bonnet gravity, Horava-Lifshitz gravity, Brans-Dicke gravity, etc [31, 32, 33, 34, 35.

Of late an infrared modification of classical gravitation was proposed, as a generalization of the 4D effective theory in the DGP model [36. The theory considers a self-interaction term of the form $(\nabla \phi)^{2} \square \phi$ in order to recover GR in high density regions. The most striking feature of the theory is that it is invariant under the Galileon shift symmetry, $\delta_{\mu} \phi \rightarrow \delta_{\mu} \phi+c_{\mu}$, in the Minkowski background. Due to this invariance the equation of motion remains a second order differential equation, preventing the introduction of extra degrees of freedom, which are usually associated with instabilities. So we assume the FRW universe in Galileon gravity model filled with the dark matter and the modified Chaplygin gas (MCG) type dark energy. In 37 the observational constraints of MCG was studied in RS II brane. In [38 the observational constraints of MCG was studied in LQC. Moreover in [39] the parameter constraints of MCG was studied in Einstein-Aether gravity. Motivated by these we set to constrain the parameters of MCG in Galileon gravity theory. The bounds on the parameters are to be obtained using the observational data analysis mechanism. The success of any dark energy or modified gravity model, depends basically on its consistency with the observational data. This is our basic motivation for the work. We reconstruct the hubble parameter $H$ using the parameters of dark energy, dark matter and modified gravity. Then we set up a comparison scenario between the reconstructed $H\left(H_{\text {theoretical }}\right)$ and the values of $H$ obtained from observational data $\left(H_{\text {observational }}\right)$. This is accomplished by the procedure of chi-square test.

The basic concepts of Galileon gravity theory are presented in section 2. The behaviour of some reconstructed cosmological parameters is studied in section 3. The observational data analysis tools in observed Hubble data (OHD) or $H(z)-z$ (Stern), OHD+BAO and OHD+BAO+CMB for $\chi^{2}$ minimum test will be studied in section 4 and we will also investigate the bounds of unknown parameters $(A, B) \&(A, C)$ of MCG dark energy by fixing other parameters. The best-fit values of the parameters are obtained by $66 \%, 90 \%$ and $99 \%$ confidence levels. The distance modulus $\mu(z)$ against redshift $z$ for our theoretical model of the MCG in Galileon gravity model for the best fit values of the parameters and the observed SNe Ia union2 data sample. Finally the paper ends with a discussion in section 5 . 


\section{Basic Equations and Solutions for MCG in Galileon Gravity Theory}

The Galileon gravity theory is described by the action [36, 40, 41, 42, 43]:

$$
S=\int d^{4} x \sqrt{-g}\left[\phi R-\frac{\omega}{\phi}(\nabla \phi)^{2}+f(\phi) \square \phi(\nabla \phi)^{2}+\mathcal{L}_{m}\right]
$$

where $\phi$ is the Galileon field and the coupling function $f(\phi)$ has dimension of length, $(\nabla \phi)^{2}=g^{\mu \nu} \nabla_{\mu} \phi \nabla_{\nu} \phi, \square \phi=$ $g^{\mu \nu} \nabla_{\mu} \nabla_{\nu} \phi$ and $\mathcal{L}_{m}$ is the matter Lagrangian. Variation of (1) with respect to the metric $g_{\mu \nu}$ gives the Einstein's equations,

$$
\begin{gathered}
G_{\mu \nu}=\frac{T_{\mu \nu}}{2 \phi}+\frac{1}{\phi}\left(\nabla_{\mu} \nabla_{\nu} \phi-g_{\mu \nu} \square \phi\right)+\frac{\omega}{\phi^{2}}\left[\nabla_{\mu} \phi \nabla_{\nu} \phi-\frac{1}{2} g_{\mu \nu}(\nabla \phi)^{2}\right] \\
-\frac{1}{\phi}\left\{\frac{1}{2} g_{\mu \nu} \nabla_{\lambda}\left[f(\phi)(\nabla \phi)^{2}\right] \nabla^{\lambda} \phi-\nabla_{\mu}\left[f(\phi)(\nabla \phi)^{2}\right] \nabla_{\nu} \phi+f(\phi) \nabla_{\mu} \phi \nabla_{\nu} \phi \square \phi\right\}
\end{gathered}
$$

For the Friedmann-Robertson-Walker background metric, the Einstein's field eqns for Galileon gravity gives,

$$
3 H^{2}=\frac{\rho}{2 \phi}-3 H I+\frac{\omega}{2} I^{2}+\phi^{2} f(\phi)\left(3 H-\frac{\alpha_{1}}{2} I\right) I^{3}
$$

and

$$
-3 H^{2}-2 \dot{H}=\frac{p}{2 \phi}+\dot{I}+I^{2}+2 H I+\frac{\omega}{2} I^{2}-\phi^{2} f(\phi)\left(\dot{I}+\frac{2+\alpha_{1}}{2} I^{2}\right) I^{2}
$$

where $H(t)=\frac{\dot{a}}{a}, I(t)=\frac{\dot{\phi}}{\phi}$ and $\alpha_{n}[\phi(t)]=\frac{d^{n} \ln f}{d \ln \phi^{n}}$

Here $\rho=\rho_{x}+\rho_{m}$ and $p=p_{x}+p_{m}$, where $\rho_{m}$ and $p_{m}$ are the energy density and pressure of the dark matter with the equation of state given by $p_{m}=w_{m} \rho_{m}$ and $\rho_{x}, p_{x}$ are respectively the energy density and pressure contribution of some dark energy. Here we consider the universe filled with Modified Chaplygin Gas (MCG). The equation of state (EOS) of MCG is given by [17]

$$
p_{x}=A \rho_{x}-\frac{B}{\rho_{x}^{\alpha}}, \quad B>0, \quad 0 \leq \alpha \leq 1
$$

We also consider the dark matter and and the dark energy are separately conserved and the conservation equations of dark matter and dark energy (MCG) are given by

$$
\dot{\rho}_{m}+3 H\left(\rho_{m}+p_{m}\right)=0
$$

and

$$
\dot{\rho}_{x}+3 H\left(\rho_{x}+p_{x}\right)=0
$$

From first conservation equation (6) we have the solution of $\rho_{m}$ as

$$
\rho_{m}=\rho_{m 0}(1+z)^{3\left(1+w_{m}\right)}
$$

From the conservation equation (7) we have the solution of the energy density as

$$
\rho_{x}=\left[\frac{B}{A+1}+C(1+z)^{3(\alpha+1)(A+1)}\right]^{\frac{1}{\alpha+1}}
$$

where $C$ is the integrating constant, $z=\frac{1}{a}-1$ is the cosmological redshift (choosing $a_{0}=1$ ) and the first constant term can be interpreted as the contribution of dark energy. So the above equation can be written as

$$
\rho_{x}=\rho_{x 0}\left[\frac{B}{(1+A) C+B}+\frac{(1+A) C}{(1+A) C+B}(1+z)^{3(\alpha+1)(A+1)}\right]^{\frac{1}{\alpha+1}}
$$

where $\rho_{x 0}$ is the present value of the dark energy density. 


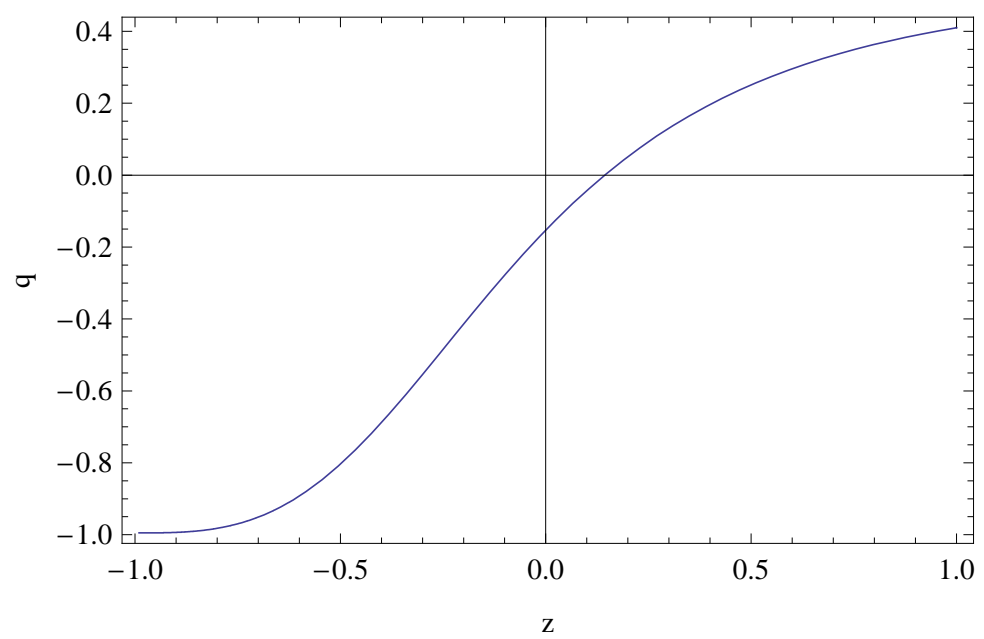

Fig.1

Fig.1 shows the variation of deceleration parameter against the redshift.

\section{Behaviour of Some Reconstructed Cosmological Parameters}

\subsection{Deceleration parameter}

From the solution of MCG (eqn. 10) and defining the dimensionless density parameters $\Omega_{m 0}=\frac{\rho_{m 0}}{3 H_{0}^{2}}$ and $\Omega_{x 0}=\frac{\rho_{x 0}}{3 H_{0}^{2}}$ and for simplicity choosing $f(\phi)=f_{0} \phi^{n}$ and $\phi=\phi_{0} a^{m}\left(f_{0}>0, \phi_{0}>0, m>0, n>0\right)$, we have the expression for Hubble parameter $H$ in terms of redshift parameter $z$ as follows (from eq. 3 ):

$$
\begin{gathered}
H(z)=\frac{1}{2\left(6 f_{0} m^{3} \phi_{0}^{3+n}-f_{0} m^{4} n \phi_{0}^{3+n}\right)}\left[-\left(m^{2} \omega-6 m-6\right)(1+z)^{m(2+n)} \phi_{0}\right. \\
+\left\{\left(\left(m^{2} \omega-6 m-6\right)(1+z)^{m(2+n)} \phi_{0}\right)^{2}-f_{0} m^{4} n \phi_{0}^{3+n}-12 H_{0}^{2}\left((1+z)^{3+m(3+n)+3 w_{m}} \Omega_{m_{0}}+\right.\right. \\
\left.\left.\left.(1+z)^{m(3+n)}\left(\frac{B}{B+(1+A) C}+\frac{(1+A) C(1+z)^{3(1+A)(1+\alpha)}}{B+(1+A) C}\right)^{\frac{1}{1+\alpha}} \Omega_{x_{0}}\right) 6 f_{0} m^{3} \phi_{0}^{3+n}\right\}^{\frac{1}{2}}\right]
\end{gathered}
$$

The study of deceleration parameter is very important for any cosmological model, considering the recent cosmic acceleration. The expression for the parameter is given by,

$$
q=-1-\frac{\dot{H}}{H^{2}}=-1+\frac{(1+z)}{H} \frac{d H}{d z}
$$

Here we reconstruct the deceleration parameter, $q$ for the model under consideration. Using equation (11) and (12), the expression for $q$ is obtained as,

$$
\begin{gathered}
q=-1+\left(( 1 + z ) \left(-12 m(2+n)(1+z)^{-1+m(2+n)} \phi_{0}-12 m^{2}(2+n)(1+z)^{-1+m(2+n)} \phi_{0}\right.\right. \\
+2 m^{3}(2+n) \omega(1+z)^{-1+m(2+n)} \phi_{0}+\left(2 m(2+n)\left(6+6 m-m^{2} \omega\right)^{2}(1+z)^{-1+2 m(2+n)} \phi_{0}^{2}\right. \\
-4 f_{0} m^{3}(-6+m n)(1+z)^{m(3+n)}\left(-3(1+z)^{3 w_{m}} \rho_{m 0}-6 z(1+z)^{3 w_{m}} \rho_{m 0}-3 z^{2}(1+z)^{3 w_{m}} \rho_{m 0}\right.
\end{gathered}
$$




$$
\begin{gathered}
-\left(m(3+n)+3 w_{m}\right)(1+z)^{-1+3 w_{m}} \rho_{m 0}-3\left(m(3+n)+3 w_{m}\right) z(1+z)^{-1+3 w_{m}} \rho_{m 0} \\
-3\left(m(3+n)+3 w_{m}\right) z^{2}(1+z)^{-1+3 w_{m}} \rho_{m 0}-\left(m(3+n)+3 w_{m}\right) z^{3}(1+z)^{-1+3 w_{m}} \rho m 0 \\
\left.\left.-\frac{m(3+n)\left(\frac{B+(1+A) C(1+z)^{3(1+A)(1+\alpha)}}{B+C+A C}\right)^{\frac{1}{1+\alpha}} \rho_{x 0}}{1+z}-\frac{3(1+A)^{2} C\left(\frac{B+(1+A) C(1+z)^{3(1+A)(1+\alpha)}}{B+C+A C}\right)^{\frac{1}{1+\alpha}} \rho_{x 0}}{(1+z)\left(C+A C+B(1+z)^{-3(1+A)(1+\alpha)}\right)}\right) \phi_{0}^{3+n}\right) \\
\left(\sqrt { } \left(\left(6+6 m-m^{2} \omega\right)^{2}(1+z)^{2 m(2+n)} \phi_{0}^{2}+4 f_{0} m^{3}(-6+m n)(1+z)^{m(3+n)}\left((1+z)^{3+3 w_{m}} \rho_{m 0}\right.\right.\right. \\
\left.\left.\left.\left.+\left(\frac{B+(1+A) C(1+z)^{3(1+A)(1+\alpha)}}{B+C+A C}\right)^{\frac{1}{1+\alpha}} \rho_{x 0}\right) \phi_{0}^{3+n}\right)\right)\right) 4\left(-6(1+z)^{m(2+n)} \phi_{0}-6 m(1+z)^{m(2+n)} \phi_{0}\right. \\
+m^{2} \omega(1+z)^{m(2+n)} \phi_{0}+\sqrt{ }\left(\left(6+6 m-m^{2} \omega\right)^{2}(1+z)^{2 m(2+n)} \phi_{0}^{2}+4 f_{0} m^{3}(-6+m n)(1+z)^{m(3+n)}\right. \\
\left.\left((1+z)^{3+3 w_{m}} \rho_{m 0}+\left(\frac{B+(1+A) C(1+z)^{3(1+A)(1+\alpha)}}{B+C+A C}\right)^{\frac{1}{1+\alpha}} \rho_{x 0} \phi_{0}^{3+n}\right)\right)
\end{gathered}
$$

\subsection{EoS parameter}

The Equation of state $(\mathrm{EoS})$ parameter, $w$ determines the nature of matter content of the universe. Its value gives an idea about the era of the universe. $w=1$ and $w=1 / 3$ predicts the stiff fluid and the radiation era respectively. $w=0$ gives the dust era. $w=-1 / 3$ corresponds to quintessence (dark energy). $w=-1$ and $w<-1$ represent the $\Lambda C D M$ and phantom era respectively. The EoS parameter can be obtained as

$$
w=\frac{2 q-1}{3}
$$

Here we reconstruct it for the given model, and plot it against the redshift parameter in fig.2.

\subsection{Statefinder parameters}

In order to distinguish between the numerous dark energy models, Sahni et al in 2003 [7] proposed a cosmological diagnostic pair $\{r, s\}$ which is known as as statefinder parameters. Since the two parameters are derived from the cosmic scale factor alone, they are dimensionless and geometrical in nature. The diagnostic pair is defined as follows:

$$
r=1+3 \frac{\dot{H}}{H^{2}}+\frac{\ddot{H}}{H^{3}} \text { and } s=\frac{r-1}{3\left(q-\frac{1}{2}\right)}
$$

Clear difference in trajectories are found when different dark energy models are examined in the $r-s$ plane, thus making the pair extremely important in the study of dark energy. Here we reconstruct the diagnostic pair for the given model using equation (11) and (15), and the simulate their nature in figs. 3,4 and 5 .

In the next section, we shall investigate some bounds of the parameters in Galileon gravity by observational data fitting. The parameters are determined by $H(z)-z$ (Stern) or OHD, OHD $+\mathrm{BAO}$ and OHD $+\mathrm{BAO}+\mathrm{CMB}$ joint data analysis [44, 45, 46]. We shall use the $\chi^{2}$ minimization technique (statistical data analysis) to get the constraints of the parameters of MCG in Galileon gravity model. 


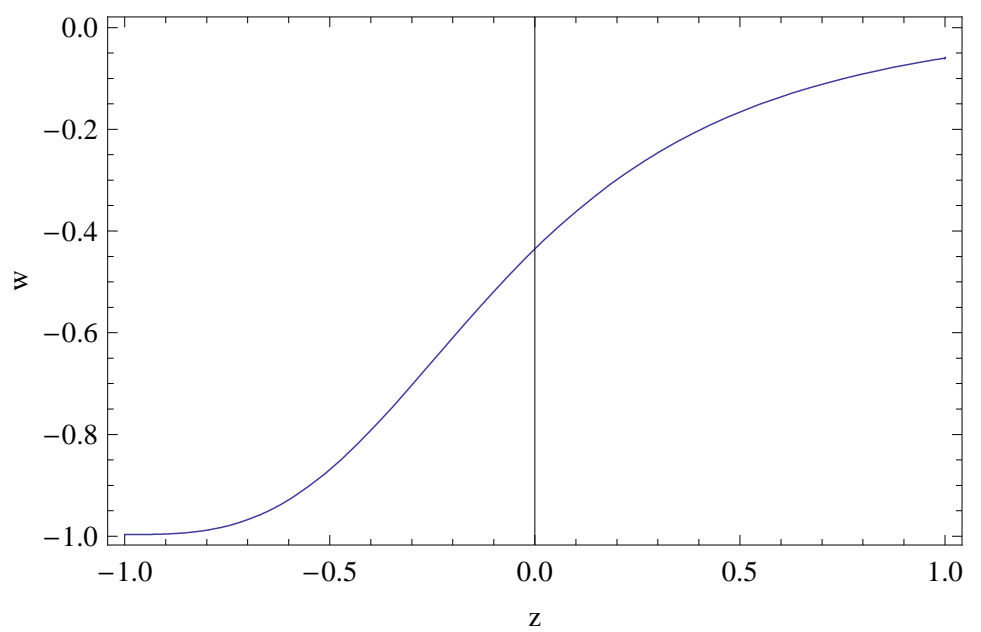

Fig.2

Fig.2 gives the plot of the EoS parameter against the redshift parameter.

\section{Observational Data Analysis}

From eqn. (11), we see that $H(z)$ contains the unknown parameters like $A, B, C, \Omega_{m 0}, \Omega_{x 0}, \alpha, n, m, \omega, w_{m}$, $f_{0}, \phi_{0}$. Now the relation between two parameters will be obtained by fixing the other parameters and by using observational data set. Eventually the bounds of the parameters will be obtained by using this observational data analysis mechanism.

\begin{tabular}{|c|c|c|}
\hline$z$ & $H(z)$ & $\sigma(z)$ \\
\hline 0 & 73 & \pm 8 \\
0.1 & 69 & \pm 12 \\
0.17 & 83 & \pm 8 \\
0.27 & 77 & \pm 14 \\
0.4 & 95 & \pm 17.4 \\
0.48 & 90 & \pm 60 \\
0.88 & 97 & \pm 40.4 \\
0.9 & 117 & \pm 23 \\
1.3 & 168 & \pm 17.4 \\
1.43 & 177 & \pm 18.2 \\
1.53 & 140 & \pm 14 \\
1.75 & 202 & \pm 40.4 \\
\hline
\end{tabular}

Table 1: The Hubble parameter $H(z)$ and the standard error $\sigma(z)$ for different values of redshift $z$.

\subsubsection{Analysis with Stern $(H(z)-z)$ Data Set}

We analyze the model, using observed value of Hubble parameter at different redshifts (twelve data points) listed in observed Hubble data by Stern et al [48. The Hubble parameter $H(z)$ and the standard error $\sigma(z)$ for different values of redshift $z$ are given in Table 1 . Since we are using testing of hypothesis, so before proceeding, we form our null and alternate hypothesis which are given below.

\section{Null Hypothesis:}

$$
H_{0}: H_{\text {theoretical }}=H_{\text {observational }}
$$




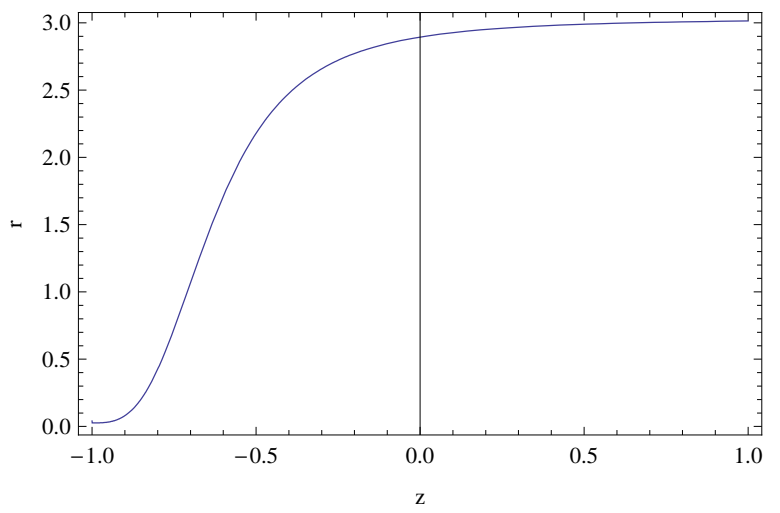

Fig.3

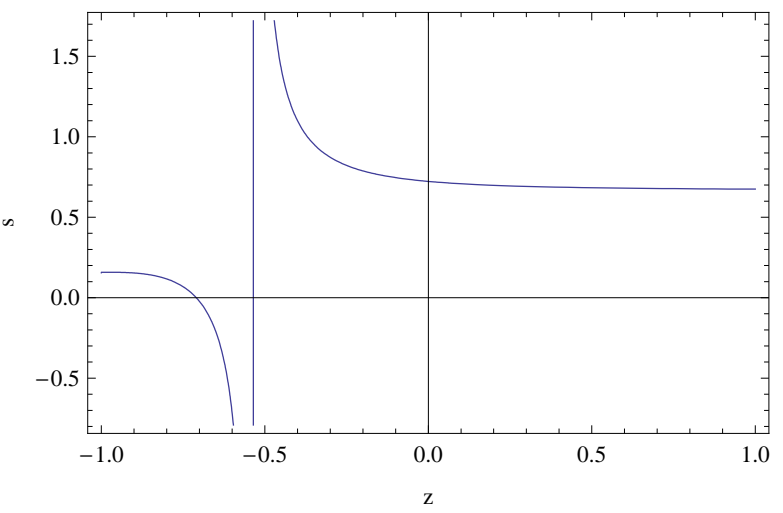

Fig.4

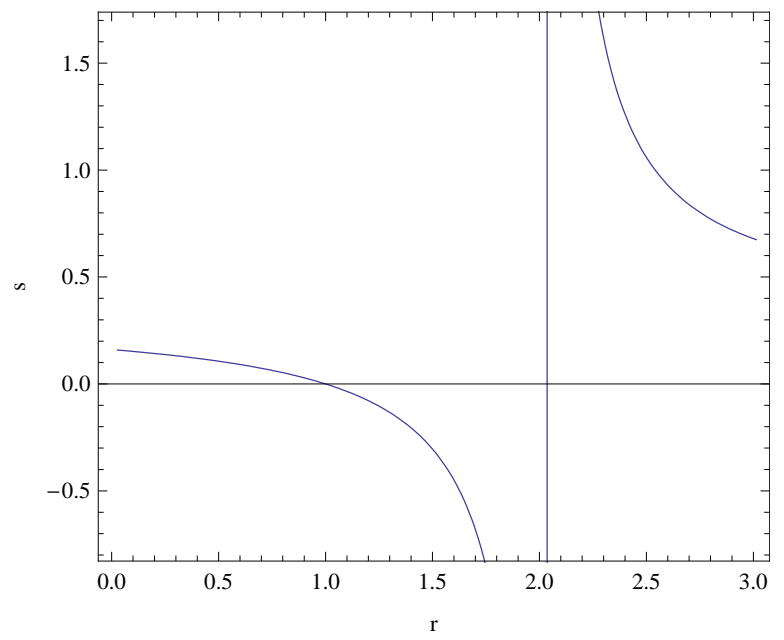

Fig.5

Fig.3-4 show the plots of statefinder parameters against redshift.

Fig. 5 gives the trajectories in $r$-s plane. 


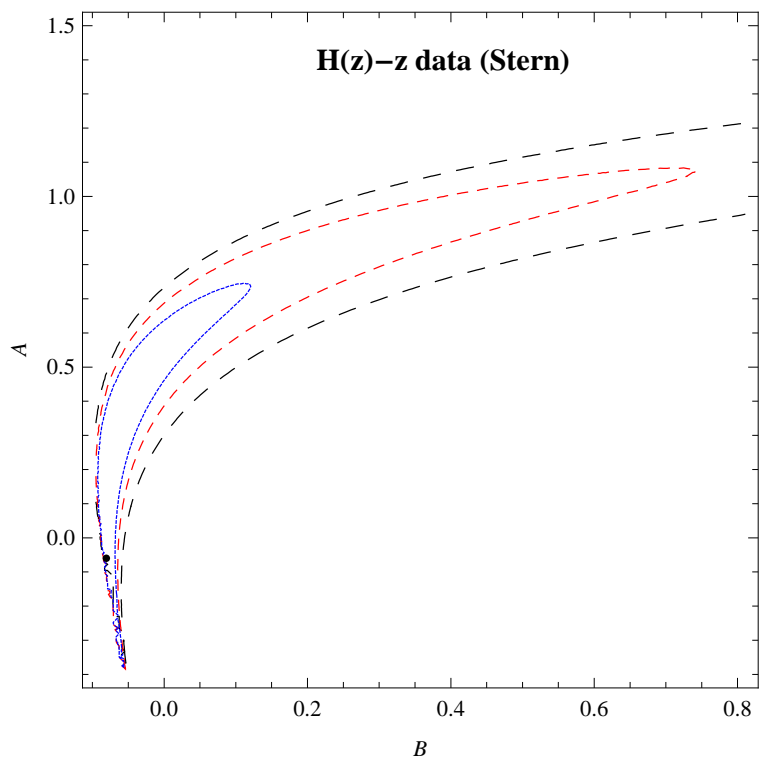

Fig.6

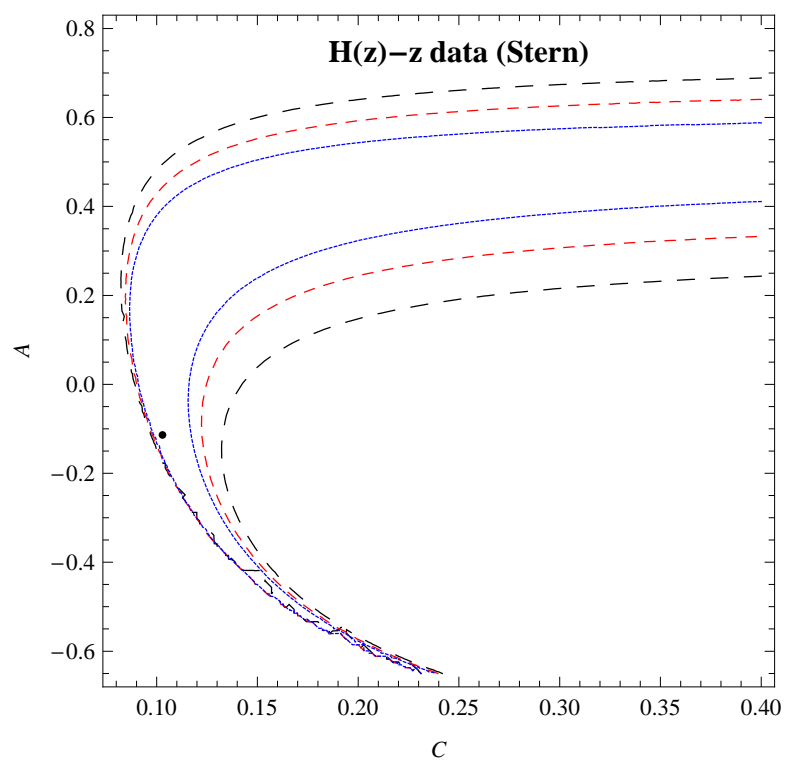

Fig.7

Fig. $6 \& 7$ show the variations of $A$ with $B$ and $A$ with $C$ respectively for different confidence levels in Galileon gravity with modified Chaplygin gas. The 66\% (solid, blue), $90 \%$ (dashed, red) and $99 \%$ (dashed, black) contours are plotted in these figures for the $H(z)-z$ (Stern) analysis.

\section{Alternative Hypothesis: $\quad H_{1}: H_{\text {theoretical }} \neq H_{\text {observational }}$}

Here we test the null hypothesis $H_{0}$ against the alternative hypothesis $H_{1}$. For this purpose we first form the $\chi^{2}$ statistics as a sum of standard normal distribution as follows:

$$
\chi_{\text {Stern }}^{2}=\sum \frac{\left(H(z)-H_{\text {obs }}(z)\right)^{2}}{\sigma^{2}(z)}
$$

where $H(z)$ and $H_{o b s}(z)$ are theoretical and observational values of Hubble parameter at different redshifts respectively. Here, $H_{o b s}$ is a nuisance parameter and can be safely marginalized. We consider the present value of Hubble parameter $H_{0}=72 \pm 8 \mathrm{Kms}^{-1} \mathrm{Mpc}^{-1}$ and a fixed prior distribution. Here we shall determine best fit value of the parameters $(A, B) \&(A, C)$ by minimizing the above distribution $\chi_{\text {Stern }}^{2}$ and fixing the other unknown parameters with the help of Stern data. We now plot the graph for different confidence levels. In early stage the Chaplygin Gas follow the equation of state $p=A \rho$ where $A \leq 1$. So, as per our theoretical model the two parameters should satisfy the two inequalities $A \leq 1$ and $B>0$. Now our best fit analysis with Stern observational data support the theoretical range of the parameters. The $66 \%$ (solid, blue), $90 \%$ (dashed, red) and $99 \%$ (dashed, black) contours are plotted in figures 6 and 7 for $\Omega_{m 0}=0.0014, \Omega_{x 0}=0.0014, \alpha=0.001$, $n=0.5, m=10, \omega=-3, w_{m}=0.03, f_{0}=0.01, \phi_{0}=0.01$. The best fit values of $(A, B) \&(A, C)$ are tabulated in Table 2.

\begin{tabular}{|c|c|c|}
\hline$A$ & $B$ & $\chi_{\min }^{2}$ \\
\hline-0.0597946 & -0.0805254 & 333.628 \\
\hline$A$ & $C$ & $\chi_{\min }^{2}$ \\
\hline-0.115326 & 0.102968 & 332.727 \\
\hline
\end{tabular}

Table 2: $H(z)-z$ (Stern): The best fit values of $A$ with $B$ and $C$ for the minimum values of $\chi^{2}$. 


\subsubsection{Joint Analysis with Stern + BAO Data Sets}

The method of joint analysis, the Baryon Acoustic Oscillation (BAO) peak parameter value has been proposed by [7] and we shall use their approach. Sloan Digital Sky Survey (SDSS) survey is one of the first redshift survey by which the BAO signal has been directly detected at a scale $\sim 100 \mathrm{MPc}$. The said analysis is actually the combination of angular diameter distance and Hubble parameter at that redshift. This analysis is independent of the measurement of $H_{0}$ and not containing any particular dark energy. Here we examine the parameters $B$ and $C$ for Chaplygin gas model from the measurements of the BAO peak for low redshift (with range $0<z<0.35$ ) using standard $\chi^{2}$ analysis. The error is corresponding to the standard deviation, where we consider Gaussian distribution. Low-redshift distance measurements is a lightly dependent on different cosmological parameters, the equation of state of dark energy and have the ability to measure the Hubble constant $H_{0}$ directly. The BAO peak parameter may be defined by

$$
\mathcal{A}=\frac{\sqrt{\Omega_{m}}}{E\left(z_{1}\right)^{1 / 3}}\left(\frac{1}{z_{1}} \int_{0}^{z_{1}} \frac{d z}{E(z)}\right)^{2 / 3}
$$

Here $E(z)=H(z) / H_{0}$ is the normalized Hubble parameter, the redshift $z_{1}=0.35$ is the typical redshift of the SDSS sample and the integration term is the dimensionless comoving distance to the to the redshift $z_{1}$ The value of the parameter $\mathcal{A}$ for the flat model of the universe is given by $\mathcal{A}=0.469 \pm 0.017$ using SDSS data [7] from luminous red galaxies survey. Now the $\chi^{2}$ function for the BAO measurement can be written as

$$
\chi_{B A O}^{2}=\frac{(\mathcal{A}-0.469)^{2}}{(0.017)^{2}}
$$

Now the total joint data analysis (Stern+BAO) for the $\chi^{2}$ function may be defined by

$$
\chi_{\text {total }}^{2}=\chi_{\text {Stern }}^{2}+\chi_{B A O}^{2}
$$

According to our analysis the joint scheme(Stern+BAO) gives the best fit values of $(A, B) \&(A, C)$ in Table 3. Finally we draw the contours for the $66 \%$ (solid,blue), $90 \%$ (dashed, red) and $99 \%$ (dashed, black) confidence limits depicted in figures 8 and 9 for $\Omega_{m 0}=0.0014, \Omega_{x 0}=0.0014, \alpha=0.001, n=0.5, m=10, \omega=-3$, $w_{m}=0.03, f_{0}=0.01, \phi_{0}=0.01$.

\begin{tabular}{|c|c|c|}
\hline$A$ & $B$ & $\chi_{\min }^{2}$ \\
\hline 1.95212 & 7.6453 & 373.061 \\
\hline$A$ & $C$ & $\chi_{\min }^{2}$ \\
\hline 2.0324 & 0.0763776 & 372.895 \\
\hline
\end{tabular}

Table 3: $H(z)-z$ (Stern) + BAO: The best fit values of $A$ with $B$ and $C$ for the minimum values of $\chi^{2}$.

\subsubsection{Joint Analysis with Stern + BAO + CMB Data Sets}

One interesting geometrical probe of dark energy can be determined by the angular scale of the first acoustic peak through angular scale of the sound horizon at the surface of last scattering which is encoded in the CMB power spectrum Cosmic Microwave Background (CMB) shift parameter is defined by [49, 50, 51]. It is not sensitive with respect to perturbations but are suitable to constrain model parameter. The CMB power spectrum first peak is the shift parameter which is given by

$$
\mathcal{R}=\sqrt{\Omega_{m}} \int_{0}^{z_{2}} \frac{d z}{E(z)}
$$

where $z_{2}$ is the value of redshift at the last scattering surface. From WMAP7 data of the work of Komatsu et al [52] the value of the parameter has obtained as $\mathcal{R}=1.726 \pm 0.018$ at the redshift $z=1091.3$. Now the $\chi^{2}$ function for the CMB measurement can be written as

$$
\chi_{C M B}^{2}=\frac{(\mathcal{R}-1.726)^{2}}{(0.018)^{2}}
$$

Now when we consider three cosmological tests together, the total joint data analysis (Stern+BAO $+\mathrm{CMB}$ ) for the $\chi^{2}$ function may be defined by 


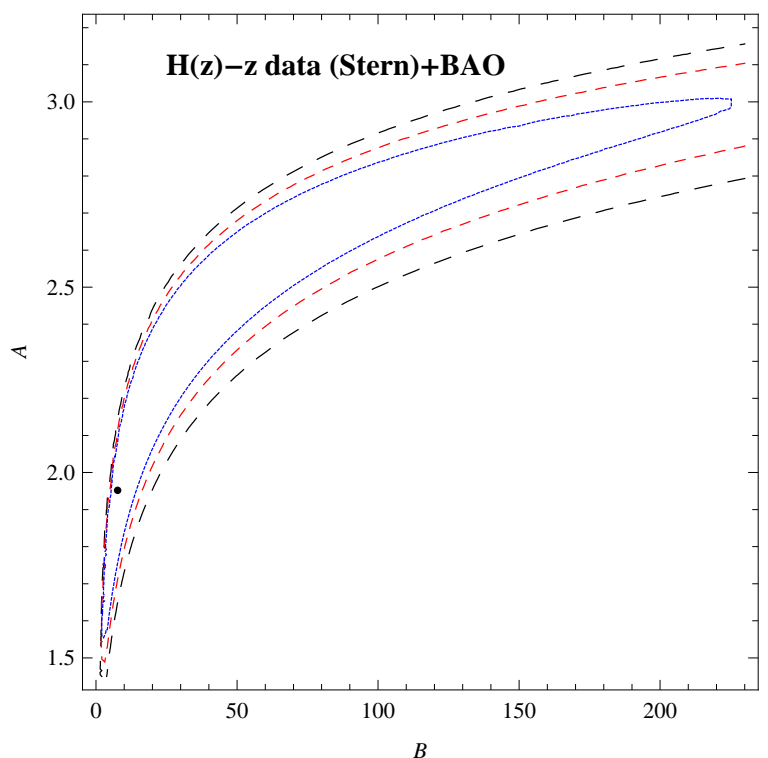

Fig.8

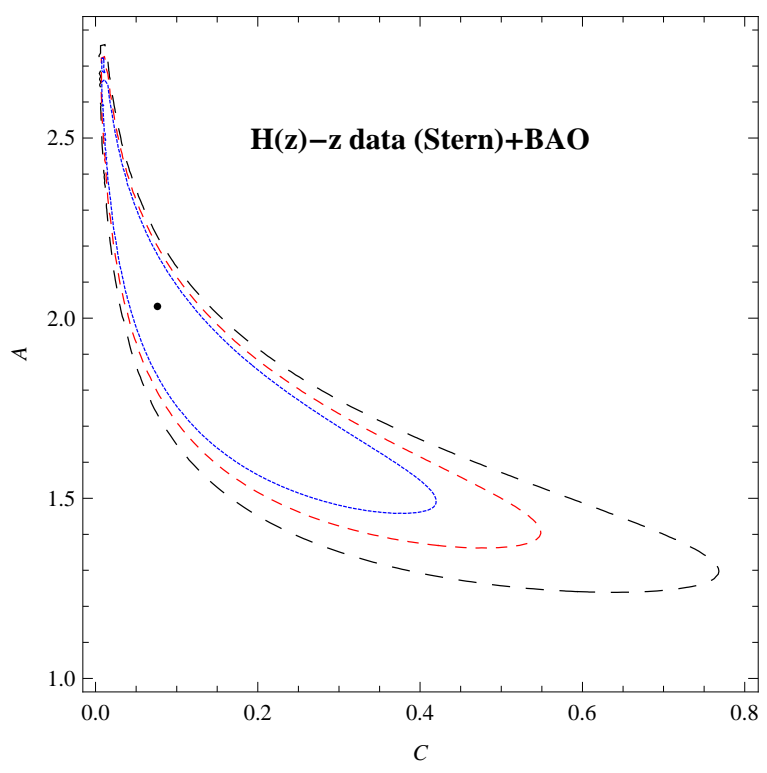

Fig.9

Fig. $8 \& 9$ show the variations of $A$ with $B$ and $A$ with $C$ respectively for different confidence levels in Galileon gravity with modified Chaplygin gas. The 66\% (solid, blue), $90 \%$ (dashed, red) and $99 \%$ (dashed, black) contours are plotted in these figures for the $H(z)-z$ (Stern) $+\mathrm{BAO}$ joint analysis.

$$
\chi_{T O T A L}^{2}=\chi_{S t e r n}^{2}+\chi_{B A O}^{2}+\chi_{C M B}^{2}
$$

Now the best fit values of $(A, B) \&(A, C)$ for joint analysis of BAO and CMB with Stern observational data support the theoretical range of the parameters given in Table 4 . The $66 \%$ (solid, blue), $90 \%$ (dashed, red) and $99 \%$ (dashed, black) contours are plotted in figures 10 and 11 for $\Omega_{m 0}=0.0014, \Omega_{x 0}=0.0014, \alpha=0.001$, $n=0.5, m=10, \omega=-3, w_{m}=0.03, f_{0}=0.01, \phi_{0}=0.01$.

\begin{tabular}{|c|c|c|}
\hline$A$ & $B$ & $\chi_{\min }^{2}$ \\
\hline-0.0099825 & -27.5635 & 10121.654 \\
\hline$A$ & $C$ & $\chi_{\min }^{2}$ \\
\hline-0.0100278 & 0.020933 & 10121.739 \\
\hline
\end{tabular}

Table 4: $H(z)-z$ (Stern) $+\mathrm{BAO}+\mathrm{CMB}$ : The best fit values of $A$ with $B$ and $C$ for the minimum values of $\chi^{2}$.

\subsubsection{Redshift-Magnitude Observations from Supernovae Type Ia}

The Supernova Type Ia experiments provided the main evidence for the existence of dark energy. Since 1995, two teams of High- $z$ Supernova Search and the Supernova Cosmology Project have discovered several type Ia supernovas at the high redshifts [1, 2, 3, 53. The observations directly measure the distance modulus of a Supernovae and its redshift $z$ [54, 55. Now, take recent observational data, including SNe Ia which consists of 557 data points and belongs to the Union2 sample [56. From the observations, the luminosity distance $d_{L}(z)$ determines the dark energy density and is defined by

$$
d_{L}(z)=(1+z) H_{0} \int_{0}^{z} \frac{d z^{\prime}}{H\left(z^{\prime}\right)}
$$

and the distance modulus (distance between absolute and apparent luminosity of a distance object) for Supernovas is given by 


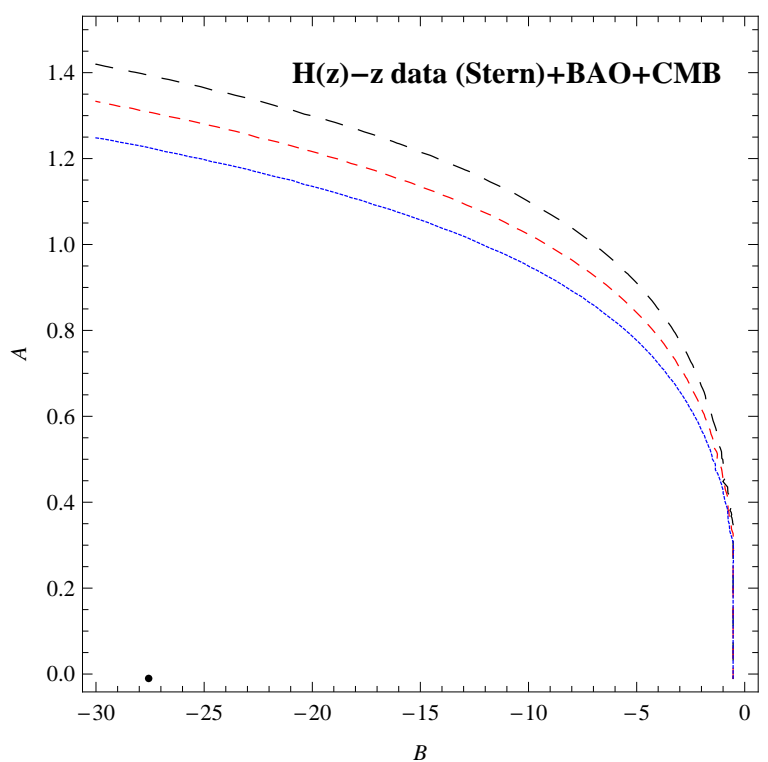

Fig.10

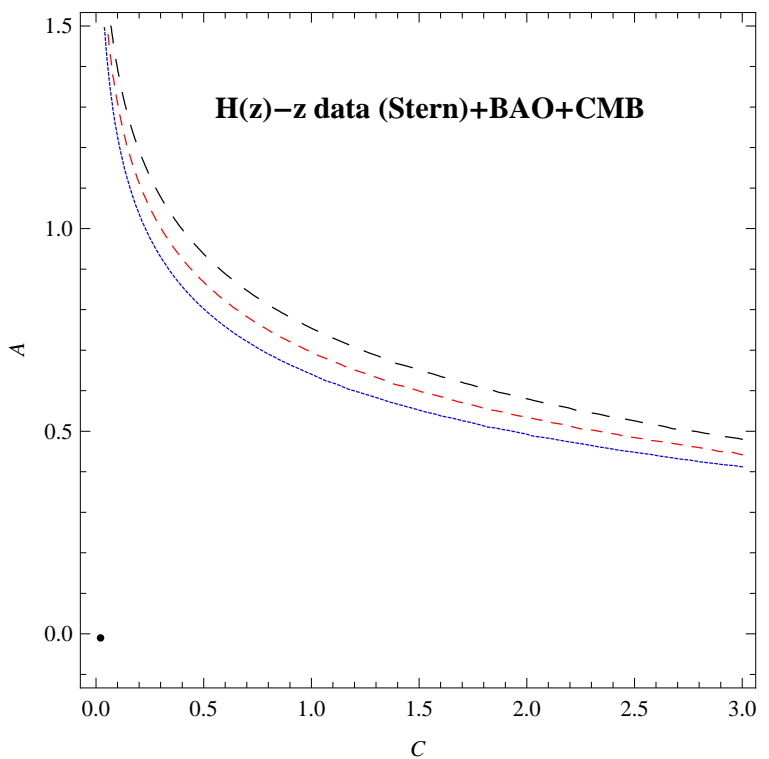

Fig.11

Fig.10 \& 11 show the variations of $A$ with $B$ and $A$ with $C$ respectively for different confidence levels in Galileon gravity with modified Chaplygin gas. The 66\% (solid, blue), $90 \%$ (dashed, red) and $99 \%$ (dashed, black) contours are plotted in these figures for the $H(z)-z$ (Stern) $+\mathrm{BAO}+\mathrm{CMB}$ joint analysis.

$$
\mu(z)=5 \log _{10}\left[\frac{d_{L}(z) / H_{0}}{1 M P c}\right]+25
$$

The best fit of distance modulus as a function $\mu(z)$ of redshift $z$ for our theoretical model and the Supernova Type Ia Union2 sample are drawn in figure 12 for our best fit values of $A, B$ and $C$ for (Stern) $+\mathrm{BAO}+\mathrm{CMB}$ joint analysis as $A=-0.010, B=-27.563, C=0.020, \Omega_{m 0}=0.0014, \Omega_{x 0}=0.0014, \alpha=0.001, n=0.5, m=10$, $\omega=-3, w_{m}=0.03, f_{0}=0.01, \phi_{0}=0.01$. From the curves, we see that the theoretical MCG model in Galileon gravity is in agreement with the union2 sample data.

\section{Discussions}

In this work, we have considered the FRW universe in Galileon gravity filled with a combination of dark matter and dark energy in the form of Modified Chaplygin gas (MCG). Since, MCG is one of the candidate of unified dark matter-dark energy model. We present the Hubble parameter in terms of the observable parameters $\Omega_{m 0}$, $\Omega_{x 0}$ and $H_{0}$ with the redshift $z$ and the other model parameters like $A, B, C, \alpha n, m, \omega, w_{m}, f_{0}$ and $\phi_{0}$. We have chosen the observed values of $f_{0}=0.01, \phi_{0}=0.01, \alpha=0.001, w_{m}=0.03, \omega=-3, n=0.5, m=10$, and $H_{0}=72 \mathrm{Mpc}^{-1}$. In figure 1, the plot of reconstructed deceleration parameter $q$ is obtained against the redshift parameter $z$. It can be seen that the present universe $(z=0)$ is undergoing a cosmic acceleration, which is evident from the negative value of $q$. From the plot it can also be predicted that this acceleration will continue late in the future universe $(z<0)$. In figure 2 , we have obtained the plot of the EoS parameter $(w)$ vs Redshift $(z)$. In the curve $z=0$ corresponds to $w=-0.5$ (approx.). Hence it is the testimony of the fact that the current epoch is dominated by dark energy and consequently accelerating in nature. It should also be noted that the curve acquires an asymptotic behavior near $z=-1$, which almost corresponds to $w=-1$. We know that physically the admissible values of the redshift parameter is $z>-1$. For present universe $z=0$ and for future universe $z<0$. In the interval $-1<z<0$, the universe continues in the accelerating phase but, the value of $w>-1$. This is true for almost any admissible values of the model parameters. So it may be concluded that 


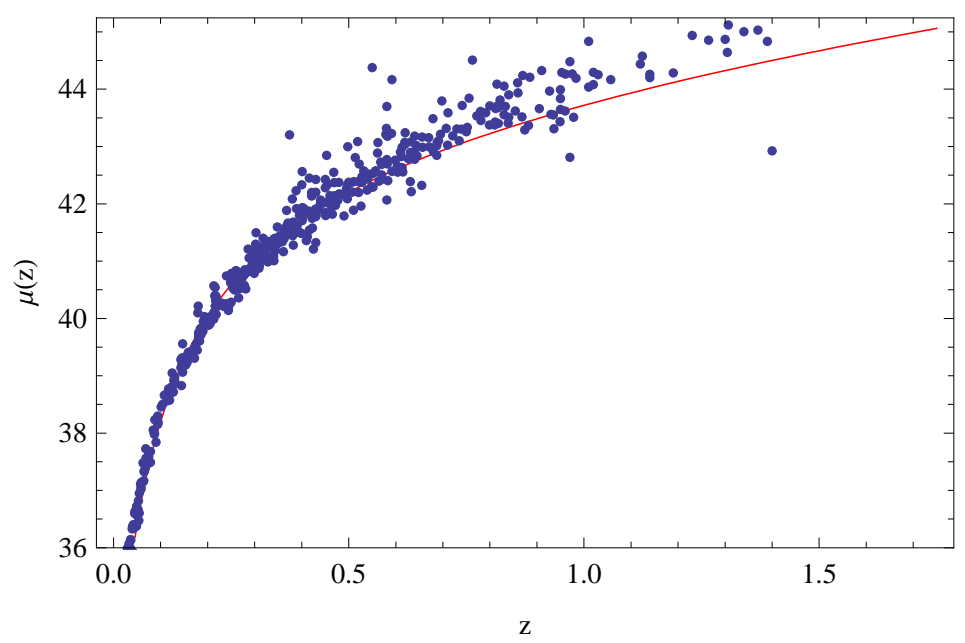

Fig.12

Fig.12 shows $\mu(z)$ vs $z$ for Galileon gravity with MCG (solid red line) and the Union2 sample (dotted points).

the reconstructed model does not go beyond $\Lambda C D M$. In figures 3 and 4 the plots of the statefinder parameters $(r, s)$ are generated against redshift. These serves the purpose of distinguishing the model under consideration with other models, presenting a unique nature to it (the model). Finally in figure 5, the trajectories in $r$ - $s$ plane is obtained. It is worth noticing that $r$ tends towards 1 as $s$ tends towards 0 . Therefore it is evident that these results tends towards the $\Lambda C D M$ model.

From Stern data set (12 points), we have obtained the bounds of the arbitrary parameters $(A, B) \&(A, C)$ by minimizing the $\chi^{2}$ test. Next due to joint analysis of Stern $+B A O$ and Stern $+B A O+C M B$ observations, we have also obtained the best fit values and the bounds of the parameters $(A, B) \&(A, C)$. We have plotted the statistical confidence contour of $(A, B) \&(A, C)$ for different confidence levels i.e., $66 \%$ (dotted, blue), $90 \%$ (dashed, red) and 99\%(dashed, black) confidence levels by fixing observable parameters $\Omega_{m 0}, \Omega_{x 0}$ and $H_{0}$ and some other parameters $\alpha, n, m, \omega, w_{m}, f_{0}$ and $\phi_{0}$ for Stern, Stern $+B A O$ and Stern $+B A O+C M B$ data analysis.

From the Stern data, the best-fit values and bounds of the parameters $(A, B) \&(A, C)$ are obtained and are shown in Table 2 and the figures $6 \& 7$ shows statistical confidence contour for $66 \%, 90 \%$ and $99 \%$ confidence levels. Next due to joint analysis with Stern $+B A O$ data, we have also obtained the best-fit values and bounds of the parameters $(A, B) \&(A, C)$ and are shown in Table 3 and in figures $8 \& 9$ we have plotted the statistical confidence contour for $66 \%, 90 \%$ and $99 \%$ confidence levels. After that, due to joint analysis with Stern $+B A O+C M B$ data, the best-fit values and bounds of the parameters $(A, B) \&(A, C)$ are found and are shown in Table 4 and the figures $10 \& 11$ shows statistical confidence contour for $66 \%, 90 \%$ and $99 \%$ confidence levels. For each case, we compare the model parameters through the values of the parameters and by the statistical contours. From this comparative study, one can understand the convergence of theoretical values of the parameters to the values of the parameters obtained from the observational data set and how it changes for different parametric values. The distance modulus $\mu(z)$ has been drawn against redshift $z$ in figure 12 for our theoretical model of the MCG in Galileon gravity for the best fit values of the parameters of Stern $+B A O+C M B$ data and the observed SNe Ia Union2 data sample.

The observational study discovers the constraint of allowed composition of matter-energy by constraining the range of the values of the parameters for a physically viable MCG in Galileon gravity model . We have also verified that when $\lambda$ is large, the best fit values of the parameters and other results of Galileon gravity model in MCG coincide with the results in Einstein's gravity [45. When $\lambda$ is small, the best fit values of the parameters and the bounds of parameters spaces in different confidence levels in Galileon gravity model can be clearly distinguished from Einstein's gravity for MCG dark energy model. From the above discussion, we can conclude that the observational data sets are perfectly consistent with our predicted theoretical MCG model in 
Galileon gravity. Finally, it is worth mentioning that, even though the quantum aspect of gravity have small effect on the observational constraint, but the cosmological observation can put upper bounds on the magnitude of the correction coming from quantum gravity that may be closer to the theoretical expectation than what one would expect.

\section{Acknowledgements}

The authors sincerely acknowledge the facilities provided by the Inter-University Centre for Astronomy and Astrophysics (IUCAA), pune, India where a part of the work was carried out. Authors also thank the anonymous referee for his/her invaluable comments that helped them to improve the quality of the manuscript.

\section{References}

[1] Perlmutter, S. J. et al :- Nature 39151 (1998).

[2] Riess, A. G. et al.[Supernova Search Team Collaboration] :- Astron. J. 1161009 (1998).

[3] Riess, A. G. et al. :- Astrophys. J. 607665 (2004).

[4] Bennet, C. et al. :- Phys. Rev. Lett. 852236 (2000).

[5] Spergel, D. N. et al. :- Astrophys. J. Suppl. Ser. 170377 (2007).

[6] Adelman-McCarthy, J. K. et al. :- Astrophys. J. Suppl. Ser. 175297 (2008).

[7] Eisenstein, D. J. et al. [SDSS Collaboration] :- Astrophys. J. 633560 (2005).

[8] Briddle, S. et al. :- Science 2991532 (2003).

[9] Spergel, D. N. et al. :- Astrophys. J. Suppl. 148, 175 (2003).

[10] Peebles, P. J. E., Ratra, B. :- Astrophys. J. 325 L17 (1988).

[11] Caldwell, R. R., Dave, R., Steinhardt, P. J. :- Phys. Rev. Lett. 801582 (1998).

[12] Armendariz - Picon, C., Mukhanov, V. F., Steinhardt, P. J. :- Phys. Rev. Lett. 854438 (2000).

[13] Sen, A. :- JHEP 0207065 (2002).

[14] Caldwell, R. R. :- Phys. Lett. B 54523 (2002).

[15] Feng, B., Wang, X. L., Zhang, X. M. :- Phys. Lett. B 60735 (2005).

[16] Kamenshchik, A. Y., Moschella, U., Pasquier, V. :- Phys. Lett. B 511265 (2001).

[17] Debnath, U., Banerjee, A. and Chakraborty, S., :- Class. Quantum Grav. 215609 (2001).

[18] Cohen, A., Kaplan, D., Nelson, A. :- Phys. Rev. Lett. 82, 4971 (1999).

[19] Sahni, V., Shtanov, Y. :- JCAP 0311014 (2003).

[20] Cai, R. G. :- Phys. Lett. B 657228 (2007).

[21] Wei, H., Cai, R. G. :- Phys. Lett. B 660113 (2008).

[22] Choudhury, T. R., Padmanabhan, T. :- Astron. Astrophys. 429807 (2007).

[23] Tonry, J. L. et al. :- Astrophys. J. 5941 (2003).

[24] Barris, B. J. et al. :- Astrophys. J. 602571 (2004).

[25] Lu, J. et al. :- Phys. Lett. B 66287 (2008).

[26] Dao-Jun, L., Xin-Zhou, L. :- Chin. Phys. Lett. 221600 (2005).

[27] Dvali, G. R., Gabadadze, G., Porrati, M. :- Phys. Lett. B 484112 (2000).

[28] De Felice, A., Tsujikawa, T. :- arXiv: 1002.4928 [gr-qc]. 
[29] S. Nojiri and S. D. Odintsov, arXiv:1011.0544 [gr-q].

[30] Clifton, T., Barrow, J. :- Phys. Rev. D 72103005 (2005).

[31] Yerzhanov, K. K. et al :- arXiv:1006.3879v1 [gr-qc] (2010).

[32] Nojiri, S., Odintsov, S. D. :- Phys. Lett. B 6311 (2005).

[33] Antoniadis, I., Rizos, J., Tamvakis, K. :- Nucl. Phys. B 415497 (1994).

[34] Horava, P. :- JHEP 0903020 (2009).

[35] Brans, C., Dicke, H. :- Phys. Rev. 124925 (1961).

[36] Nicolis, A., Rattazzi, R., Trincherini, E. :- Phys. Rev. D $79064036(2009)$

[37] Ranjit, C., Rudra, P., Kundu, S. :- Astrophys. Space Sci. 347 423(2013)

[38] Chakraborty, S., Debnath, U., Ranjit, C. :- Eur. Phys. J. C. 72 2101(2012)

[39] Debnath, U. :- arXiv:1310.2144 [gr-qc] (2013)

[40] Deffayet, C., Esposito-Farese, G., Vikman, A. :- Phys. Rev. D 79084003 (2009)

[41] Deffayet, C., Deser, S., Esposito-Farese, G. :- Phys. Rev. D 80064015 (2009)

[42] Chow, N., Khoury, J. :- Phys. Rev. D 80024037 (2009)

[43] Silva, F. P., Koyama, K. :- Phys. Rev. D 80121301 (2009)

[44] Wu, P. and Yu, H. :- Phys. Lett. B 644 16(2007)

[45] Thakur, P., Ghose, S. and Paul, B. C.:- Mon. Not. R. Astron. Soc. 3971935 (2009)

[46] Paul, B. C., Ghose, S. and Thakur, P., arXiv:1101.1360v1 [astro-ph.CO].

[47] Sahni, V. et al.:- JETP 77, 201(2003).

[48] Stern, D. et al :- JCAP 1002008 (2010).

[49] Bond, J. R. et al :- Mon. Not. Roy. Astron. Soc. 291 L33 (1997)

[50] Efstathiou, G., Bond, J. R. :- Mon. Not. R. Astro. Soc. 30475 (1999)

[51] Nessaeris, S., Perivolaropoulos, L. :- JCAP 0701018 (2007).

[52] Komatsu, E. et al. :- Astrophys. J. Suppl. 19218 (2011).

[53] Perlmutter, S. J. et al :- Astrophys. J. 517565 (1999).

[54] Riess, A. G. et al. :- Astrophys. J. 65998 (2007).

[55] Kowalaski et al. :- Astrophys. J. 686749 (2008).

[56] Amanullah, R. et al. :- Astrophys. J. 716712 (2010). 